\title{
Direct Measurement Results of the Time Lag of LOS-Velocity Oscillations Between Two Heights in Solar Faculae and Sunspots
}

\author{
N. Kobanov, D. Kolobov, A. Kustov, S. Chupin, \\ and A. Chelpanov \\ Institute of Solar-Terrestrial Physics \\ of Siberian Branch of Russian Academy of Sciences, Irkutsk, Russia \\ email: kolobov@iszf.irk.ru \\ This article was firstly published in Solar Physics
}

\begin{abstract}
We present an investigation of line-of-sight (LOS) velocity oscillations in solar faculae and sunspots. To study the phase relations between chromospheric and photospheric oscillations of the LOS velocity, we measured the time lag of the chromospheric signal relative to the photospheric one for several faculae and sunspots in a set of spectral line pairs. The measured time lags are different for different objects. The mean measured delay between the oscillations in the five-minute band in faculae is 50 s for the Si I $10827 \AA$ - He I $10830 \AA$ pair; for the pair Fe I $6569 \AA-\mathrm{H} \alpha 6563 \AA$ the mean delay is $20 \mathrm{~s}$; for the pair Fe I $4551 \AA$ - Ba II $4554 \AA$ the mean delay is $7 \mathrm{~s}$; for the pair Si I $8536 \AA$ - Ca II $8542 \AA$ the mean delay is 20 s. For the oscillations in the three-minute band in sunspot umbrae the mean delay is $55 \mathrm{~s}$ for the Si I $10827 \AA$ - He I $10830 \AA$ pair; for the Fe I $6569 \AA-\mathrm{H} \alpha 6563 \AA$ pair it was not possible to determine the delay; for the Fe I $4551 \AA$ - Ba II $4554 \AA$ pair the mean delay is 6 s; for the Si I $8536 \AA$ - Ca II $8542 \AA$ pair the mean delay is $21 \mathrm{~s}$. Measured delays correspond to the wave propagation speed which significantly exceeds the generally adopted speed of sound in the photosphere. This raises the question of the origin of these oscillations. The possibility that we deal with slow MHD waves is not ruled out.
\end{abstract}




\section{Introduction}

Solar faculae and sunspots can play an important role in the energy exchange among different layers of the solar atmosphere. The oscillatory behaviour of the chromosphere in these regions is a well-established phenomenon. The first investigations into wave propagation, including studies of waves propagating in a vertical direction from the photosphere to the chromosphere, are date from the early 1970s (Beckers and Tallant, 1969; Giovanelli, 1972; Zirin and Stein, 1972; Moore and Tang, 1975; Giovanelli, Harvey, and Livingston, 1978; Lites and Chipman, 1979; Gurman et al., 1982; Lites, 1986; Gurman, 1987). But the interest in this theme is still considerable today (Georgakilas, Christopoulou, and Koutchmy, 2000, Christopoulou, Georgakilas, and Koutchmy, 2000; Rouppe van der Voort et al., 2003; Balthasar, 2003; Kobanov, Kolobov, and Makarchik, 2006; Nagashima et al., 2007; Bloomfield, Lagg, and Solanki, 2007; Balthasar and Muglach, 2010; Reznikova et al., 2012; Sych et al., 2012). The most reliable evidence of upward propagating waves is to directly measure time delays between the photospheric and chromospheric Doppler velocity oscillations. Therefore, three-minute oscillations in sunspots and five-minute oscillations the faculae are particularly interesting. The main goal of this article is to directly measure the time delay for various pairs of spectral lines.

We selected measurements with long time series, high cadence, a good signal-to-noise ratio, and a suitable object location. The latter implies, for example, that data sets were taken close to the solar disc centre to avoid projection effects. It is reasonable to observe sunspots of regular form to facilitate the interpretation and data reduction. The choice of spectral line pairs is very importance as well. For reliability, the analysis is to cover at least several objects.

\section{Observations and Data}

The observational spectral data analysed here were obtained with the Horizontal Solar Telescope at the Sayan Solar Observatory (Kobanov et al., 2009). The telescope is elevated $6 \mathrm{~m}$ above the ground, is windproof, and equipped

with a dedicated system to suppress atmospheric turbulence (Hammerschlag and Zwaan, 1973). The instrument mirrors are of $80 \mathrm{~cm}$ diameter. The focal length of the main mirror is $20 \mathrm{~m}$, hence the possible spatial resolution is $0.2^{\prime \prime}$. 
However, due to the effects of the Earth's atmosphere, the real resolution is about $1^{\prime \prime}-1.5^{\prime \prime}$. The guiding system performs targeting and object capturing with a precision of at least $1^{\prime \prime}$, and compensates for the rotation of the Sun - the target is fixed on the spectrograph's slit during the experiment. The image jitter on the slit depends on the observational conditions and can reach $2^{\prime \prime}$ on average in the series we investigated here. Some of the time series were accompanied by an overview scan of the area under investigation. The focal length of the spectrograph is $7 \mathrm{~m}$, and the diffraction grating parameters are $200 \times 300 \mathrm{~mm}, 600$ grooves per $\mathrm{mm}$.

We made observations in pairs of the following lines: Si I $10827 \AA$ and He I $10830 \AA$; Si I $8536 \AA$ and Ca II $8542 \AA$; Fe I $6569 \AA$ and $\mathrm{H} \alpha 6563 \AA$; Fe I $4551 \AA$ and Ba II $4554 \AA$. The data contain 14 time series in faculae and 17 time series in sunspots (see Tables 1 and 2). Two digital cameras were used in the observations: Princeton Instruments (PI) RTE/CCD 256H $(256 \times 1024$, visible - near infrared range), and FLIR SC-2200 (256×320, infrared range). One PI camera pixel corresponds to $0.23^{\prime \prime}$, and the FLIR camera pixel corresponds to $0.3^{\prime \prime}$. The spectral resolution of the PI camera for the Fe I $6569 \AA$ and $\mathrm{H} \alpha 6563 \AA$ lines is $8 \mathrm{m \AA}$ per pixel; for Si I $10827 \AA-\mathrm{He}$ I $10830 \AA$ it is $20 \mathrm{~m} \AA$ per pixel. The resolution of the FLIR camera is $25 \mathrm{~m} \AA$ per pixel for the Si I $10827 \AA$ - He I $10830 \AA$ pair.

For three time series (Table 2, Nos. 15-17) the following polarisation optics were used: FLC modulator (DisplayTech), quarter-wavelength plate and Nicol prism. This allows one to register two frames $I+V, I-V$ for the whole spectrum domain that is captured by the FLIR camera. The optical scheme is described in Kolobov, Kobanov, and Grigoryev (2008). The single-frame exposure time is $39 \mathrm{~ms}$ ( $25 \mathrm{~Hz}$ cadence). When using two frames obtained in $80 \mathrm{~ms}$ no object displacement along the slit was revealed. Ten even and odd frames, with respect to the modulation state, were averaged to form two frames $(I+V, I-V)$ with a $0.8 \mathrm{~s}$ total acquisition time. For the other time series, taken without polarisation optics, the cadence varies from 0.5 to $10 \mathrm{~s}$, and the total duration ranges from 42 to 198 minutes.

Processing spectrograms included standard procedures: subtraction of dark frame and flat-field correction. We obtained line-of-sight (LOS) velocity signals via different techniques: $i$ ) the Doppler compensation technique or, in other words, the lambda-meter Nikulin, Severny, and Stepanov, 1958; Rayrole, 1967), and ii) tracking of the Stokes-V profile zero-crossing position. In the lambda-meter technique, two virtual slits were used to determine the spectral position of a chosen line. The distance between the slits was set and 
remained constant during the measurements. Initially, the slits were placed at equal distances from the line centre, and hence the measured intensities are equal. If in the next spectrogram the line shifted, the intensities changed. By displacing the slits to make their intensities equal, one can determine the new location of the line. The Stokes-V zero-crossing was calculated as a mean value of the positions of the red and blue lobes for a chosen spectral line. The Stokes-V data are available only for sunspots series Nos. 15-17 (Table 2).

To analyse oscillations of a specific frequency, we used band filtration with a sixth-order Morlet wavelet. The filtration frequency range for both the photospheric and chromospheric signals was selected based on the fast Fourier transform (FFT) power spectra, obtained for different positions along the slit. We chose the position so that both signals contained peaks in the same frequency band. We used the algorithm described by Torrence and Compo (1998) to derive the FFT power spectra $\left(1 / \sigma^{2}\right.$ normalisation, where $\sigma^{2}$ is the variance of a time series), to wavelet-filter the daya and estimate the statistical significance. The power of the chromospheric and photospheric five-minute oscillations of the LOS velocity in faculae significantly exceeds the noise level. Oscillations in this band reveal themselves clearly in the original (unfiltered) signals. In practice, one does not need to check the statistical significance of the FFT power at these periods. The same is true for the chromospheric three-minute band oscillations in sunspot umbrae of the time series. Estimating the statistical significance was useful for the photospheric umbral oscillations, which are suppressed relative to the surrounding regions and might have amplitudes that are below or around the noise level. The series demonstrated a corresponding FFT power at about the $3 \sigma^{2}$ level. Here it was not possible to obtain an unambiguous phase delay between the photosphere and the chromosphere.

The He I $10830 \AA$ line profile depth in faculae sharply increases (Figure 1 (a)) compared to the surrounding area. According to our observations, the increase in different faculae varies from 2 to 5 . We used this feature of the He I $10830 \AA$ line to precisely point at faculae near the disc centre. At first, we chose an object from full-disc images in the Ca II H line and $1700 \AA$ (SDO/AIA). Then we slowly scanned the chosen region and corrected its position along the spectrograph slit, using the live image from the camera and taking the maximum depth of the He I $10830 \AA$ line into consideration. Figure 2 presents a spatial scan example for time series No. 8. Finally, the He I $10830 \AA$ intensity space-time diagrams can be used to control the posi- 
Table 1: Facula data sets, lags between five-minute waves observed in two spectral lines

\begin{tabular}{|c|c|c|c|c|c|c|}
\hline No. & Date & $\begin{array}{c}\text { Disc } \\
\text { location }\end{array}$ & $\begin{array}{l}\mathrm{T},{ }^{1} \\
\mathrm{~min}\end{array}$ & $\begin{array}{l}\mathrm{t},{ }^{2} \\
\text { sec }\end{array}$ & $\begin{array}{l}\text { Lag, } \\
\text { sec }\end{array}$ & Lines \\
\hline 1 & 17 Jul 2010 & $19^{\circ} \mathrm{N} 28^{\circ} \mathrm{W}$ & 150 & 3 & $78-100$ & Si I-He I \\
\hline 2 & 04 Aug 2010 & $15^{\circ} \mathrm{N} 05^{\circ} \mathrm{W}$ & 81 & 4 & $-12-0$ & Si I-He I \\
\hline 3 & 05 Aug 2010 & $20^{\circ} \mathrm{N} 14^{\circ} \mathrm{W}$ & 96 & 3.5 & $20-40$ & Si I-He I \\
\hline 4 & 09 Aug 2010 & $17^{\circ} \mathrm{N} 23^{\circ} \mathrm{E}$ & 136 & 3 & $40-87$ & $\mathrm{Si}$ I-He I \\
\hline 5 & 09 Aug 2010 & $18^{\circ} \mathrm{N} 22^{\circ} \mathrm{E}$ & 98 & 4 & $10-50$ & Si I-He I \\
\hline 6 & 09 Aug 2010 & $15^{\circ} \mathrm{N} 12^{\circ} \mathrm{E}$ & 68 & 3 & $68-77$ & $\mathrm{Si}$ I-He I \\
\hline 7 & 14 Aug 2010 & $13^{\circ} \mathrm{N} 05^{\circ} \mathrm{W}$ & 198 & 3 & $30-90$ & $\mathrm{Si}$ I-He I \\
\hline 8 & 15 Aug 2010 & $24^{\circ} \mathrm{N} 18^{\circ} \mathrm{E}$ & 102 & 3 & $33-39$ & Si I-He I \\
\hline 9 & 16 Aug 2010 & $32^{\circ} \mathrm{N} 00^{\circ} \mathrm{E}$ & 55 & 3 & $68-78$ & Si I-He I \\
\hline 10 & 01 Jul 2003 & $04^{\circ} \mathrm{N} 33^{\circ} \mathrm{E}$ & 42 & 10 & $-20-10$ & $\mathrm{Fe} \mathrm{I}-\mathrm{H} \alpha$ \\
\hline 11 & 18 Aug 2004 & $09^{\circ} \mathrm{N} 17^{\circ} \mathrm{E}$ & 42 & 1 & $-8-20$ & $\mathrm{Fe} \mathrm{I}-\mathrm{H} \alpha$ \\
\hline 12 & 06 Jul 2010 & $19^{\circ} \mathrm{N} 07^{\circ} \mathrm{W}$ & 92 & 2.5 & $34-71$ & $\mathrm{Fe} \mathrm{I}-\mathrm{H} \alpha$ \\
\hline 13 & 17 Aug 2005 & $20^{\circ} \mathrm{S} 30^{\circ} \mathrm{E}$ & 93 & 2 & $4-10$ & $\mathrm{Fe} \mathrm{I}-\mathrm{Ba} \mathrm{Il}$ \\
\hline 14 & 17 Aug 2005 & $20^{\circ} \mathrm{S} 26^{\circ} \mathrm{E}$ & 88 & 2 & $-6-48$ & Si I - Ca II \\
\hline
\end{tabular}

${ }^{1}$ total duration

${ }^{2}$ sampling time (cadence)

tion of the observed object in the spectrograph slit throughout a time series (Figure 1(b)). 
Table 2: Sunspot data sets, lags between three-minute waves observed in two spectral lines

\begin{tabular}{|c|c|c|c|c|c|c|c|}
\hline No. & NOAA & Date & $\begin{array}{c}\text { Disc } \\
\text { location }\end{array}$ & $\begin{array}{l}\mathrm{T},{ }^{1} \\
\min \end{array}$ & $\begin{array}{l}\mathrm{t},{ }^{2} \\
\mathrm{sec}\end{array}$ & $\begin{array}{l}\text { Lag, } \\
\text { sec }\end{array}$ & Lines \\
\hline 15 & 11251 & 18 Jul 2011 & $16^{\circ} \mathrm{N} 10^{\circ} \mathrm{W}$ & 64 & 0.8 & $64-106$ & Si I - He I \\
\hline 16 & 11251 & 18 Jul 2011 & $16^{\circ} \mathrm{N} 10^{\circ} \mathrm{W}$ & 52 & 0.8 & $20-64$ & Si I - He I \\
\hline 17 & 11251 & 19 Jul 2011 & $16^{\circ} \mathrm{N} 20^{\circ} \mathrm{W}$ & 87 & 0.8 & $-20-110$ & $\mathrm{Si}$ I-He I \\
\hline 18 & 11180 & 3 Apr 2011 & $24^{\circ} \mathrm{N} 37^{\circ} \mathrm{W}$ & 67 & 5.5 & 35 & $\mathrm{Si}$ I-He I \\
\hline 19 & 10613 & 20 May 2004 & $09^{\circ} \mathrm{S} 03^{\circ} \mathrm{W}$ & 60 & 5 & - & $\mathrm{Fe} \mathrm{I}-\mathrm{H} \alpha$ \\
\hline 20 & 10661 & 18 Aug 2004 & $07^{\circ} \mathrm{N} 19^{\circ} \mathrm{E}$ & 42 & 1 & - & $\mathrm{Fe} \mathrm{I}-\mathrm{H} \alpha$ \\
\hline 21 & 10791 & $27 \mathrm{Jul} 2005$ & $13^{\circ} \mathrm{N} 02^{\circ} \mathrm{E}$ & 100 & 2 & - & $\mathrm{Fe} \mathrm{I}-\mathrm{H} \alpha$ \\
\hline 22 & 10791 & $27 \mathrm{Jul} 2005$ & $13^{\circ} \mathrm{N} 02^{\circ} \mathrm{E}$ & 100 & 2 & - & $\mathrm{Fe} \mathrm{I}-\mathrm{H} \alpha$ \\
\hline 23 & 10794 & 5 Aug 2005 & $11^{\circ} \mathrm{S} 16^{\circ} \mathrm{E}$ & 66 & 1 & - & $\mathrm{Fe} \mathrm{I}-\mathrm{H} \alpha$ \\
\hline 24 & 10810 & 21 Sep 2005 & $11^{\circ} \mathrm{N} 30^{\circ} \mathrm{E}$ & 108 & 2 & - & $\mathrm{Fe} \mathrm{I}-\mathrm{H} \alpha$ \\
\hline 25 & 10810 & 23 Sep 2005 & $08^{\circ} \mathrm{N} 0^{\circ} \mathrm{W}$ & 73 & 10 & - & $\mathrm{Fe} \mathrm{I}-\mathrm{H} \alpha$ \\
\hline 26 & 10657 & 13 Aug 2004 & $12^{\circ} \mathrm{N} 5^{\circ} \mathrm{W}$ & 58 & 5 & $-10-20$ & $\mathrm{Fe} I-\mathrm{Ba} \mathrm{I}$ \\
\hline 27 & 10661 & 17 Aug 2004 & $09^{\circ} \mathrm{N} 24^{\circ} \mathrm{E}$ & 42 & 0.5 & $0-22$ & $\mathrm{Fe} I-\mathrm{Ba} \mathrm{I}$ \\
\hline 28 & 10661 & 18 Aug 2004 & $09^{\circ} \mathrm{N} 13^{\circ} \mathrm{E}$ & 41 & 1 & $-10-13$ & $\mathrm{Fe} I-\mathrm{Ba} \mathrm{I}$ \\
\hline 29 & 10886 & 26 May 2006 & $08^{\circ} \mathrm{N} 10^{\circ} \mathrm{E}$ & 67 & 2 & $-12-54$ & Si I-Ca II \\
\hline 30 & 10905 & 28 Aug 2006 & $06^{\circ} \mathrm{S} 10^{\circ} \mathrm{W}$ & 27 & 0.5 & - & Si I-Ca II \\
\hline 31 & 10908 & 11 Sep 2006 & $14^{\circ} \mathrm{S} 09^{\circ} \mathrm{E}$ & 25 & 0.5 & - & $\mathrm{Si}$ I-Ca II \\
\hline
\end{tabular}

${ }^{1}$ total duration

${ }^{2}$ sampling time (cadence) 
Table 3: Spectral line formation heights

\begin{tabular}{|c|c|c|c|}
\hline Line & $\begin{array}{c}\text { Height, } \\
\text { Mm }\end{array}$ & Atmosphere model & Reference \\
\hline He I $10830 \AA$ & $\sim 2$ & FALC & Avrett et al., 1994 \\
\hline Si I $10827 \AA$ & 0.3 & model-M & Bard and Carlsson (2008) \\
\hline Si I $10827 \AA$ & 0.5 & FALC & 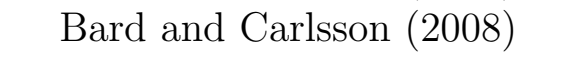 \\
\hline Ba II $4554 \AA$ & 0.64 & 3D MHD & Shchukina et al., 2009 \\
\hline Fe I $4551 \AA$ & 0.14 & HOLMU & Gurtovenko and Kostyk (1989) \\
\hline Ca II $8542 \AA$ & $1.2-1.5$ & HSRA & Mein and Mein (1980) \\
\hline Ca II $8542 \AA$ & $1.0-1.5$ & 3D MHD & Leenaarts et al. $(\overline{2009})$ \\
\hline $\mathrm{H} \alpha 6563 \AA$ & $1.5-2$ & VAL & Vernazza et al., 1981 \\
\hline $\mathrm{H} \alpha 6563 \AA$ & $1.0-1.6$ & 3D MHD & Carlsson et al., 2012 \\
\hline Fe I $6569 \AA$ & 0.15 & Bilderberg & Parnell and Beckers (1969) \\
\hline
\end{tabular}
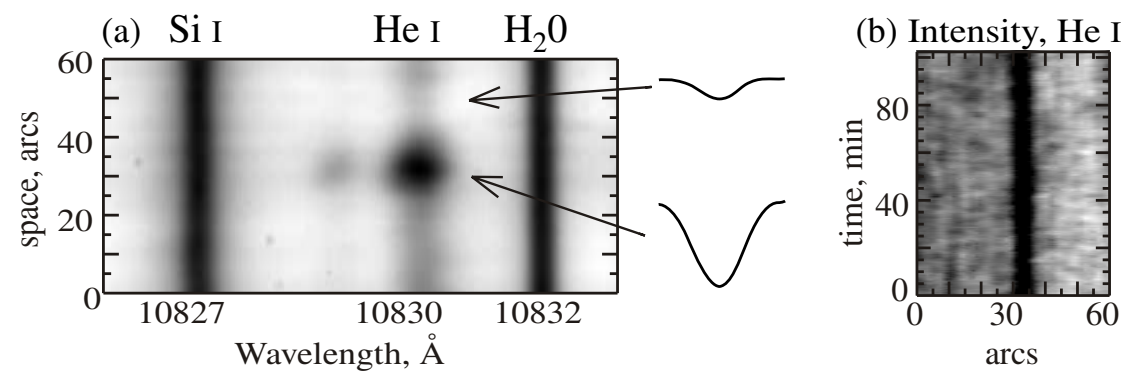

Figure 1: Time series No. 8. Overview of the data. Panel (a): spectrum for He I $10830 \AA$ demonstrating the increase in line depth in faculae; panel (b): He I $10830 \AA$ line intensity.

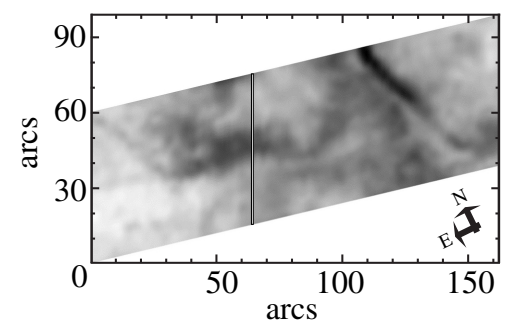

Figure 2: Spatial scan for the faculae (time series No. 8). The vertical line marks the slit position. 


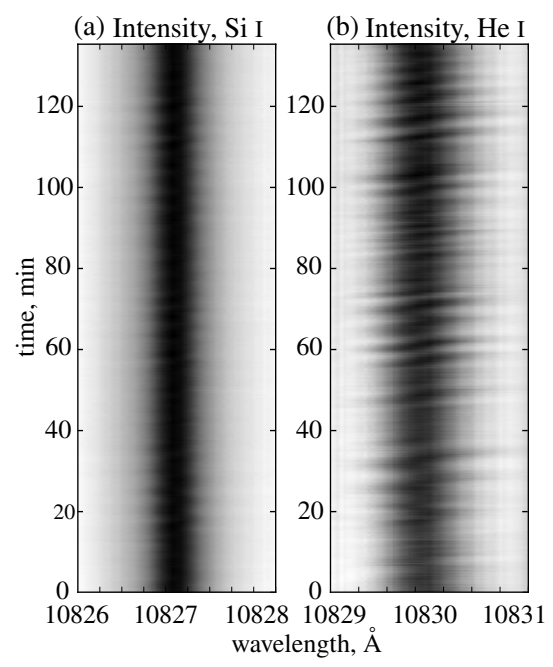

Figure 3: Time series No.4. Temporal intensity evolution for the (a) Si I $10827 \AA$ and (b) He I $10830 \AA$ lines.

\section{Measurements of the Time Delays Between LOS-velocity oscillations at Different Heights of the Solar Atmosphere}

\subsection{Faculae}

Figure 3 demonstrates the quality of the data. Table 1 presents the details about the time series and the measurement results for the time lag of the chromospheric LOS velocity relative to the photospheric velocity. The spatial coherency of the LOS-velocity oscillations in our measurements is at least about $2^{\prime \prime}$. The five-minute band oscillation power spectra often present a fine structure. At first, we determined frequency peaks that dominate both in the photosphere and in the chromosphere. Then the LOS-velocity signals, averaged over $2^{\prime \prime}$, were subjected to frequency filtration in the narrow band, usually $1 \mathrm{mHz}$, centred at the determined frequency peak. The mean amplitude of the whole photospheric signal series was equalised to match the chromospheric signal. The lag between the signals was determined using cross-correlation and was confirmed visually. As can be seen from Table 1 , the LOS-velocity five-minute oscillation chromospheric signal lags behind the photospheric signal for most of the faculae. The time lag generally does not 


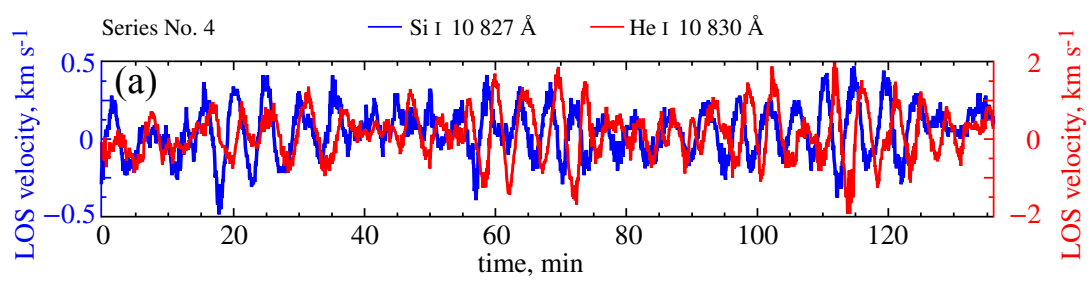

Figure 4: Time series No. 4. Original (unfiltered) LOS-velocity signals: facular photosphere (Si I $10827 \AA$, blue line) and chromosphere (He I $10830 \AA$, red line).
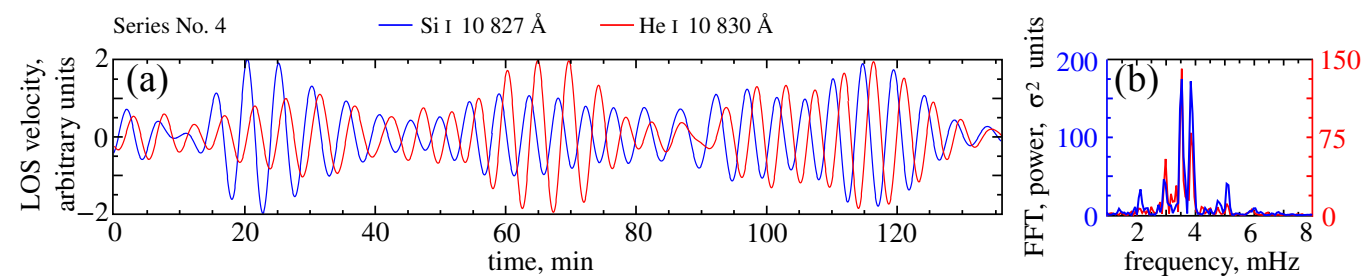

Figure 5: Time series No. 4. (a) Filtered LOS-velocity signals: facular photosphere (Si I $10827 \AA$, blue line) and chromosphere (He I $10830 \AA$, red line). $3-4 \mathrm{mHz}$ filtration band. (b) Power spectrum for the LOS-velocity signals at two height levels.

exceed $100 \mathrm{~s}$. A negative lag (downward-propagating wave) was detected for some of the time series (e.g. No. 2). Waves propagating downward were reported by Finsterle et al. (2004), McAteer et al. (2003). The corresponding wave packets at two levels can differ in detail, and different wave trains can reveal different lags.

Figure 4 presents the LOS velocity variations for a single position along the slit. The forms of the photospheric and chromospheric signals are very similar. Five-minute oscillations forming three wave trains are visible at both levels. These signals were filtered to determine the time lag more reliably (Figure 5). The frequency filter pass band was set to be $1 \mathrm{mHz}$, centred at $3.5 \mathrm{mHz}$. Narrowing the pass band did not significantly affect the structure of the profiles. After the filtration the wave trains became more pronounced. At the same time, this revealed noticeable differences in the oscillations within a single train between the photosphere and the chromosphere. The crosscorrelation between the photospheric and chromospheric signals is highest (0.97) for an $87 \mathrm{~s} \mathrm{lag}$. This value corresponds to the single spatial domain of 


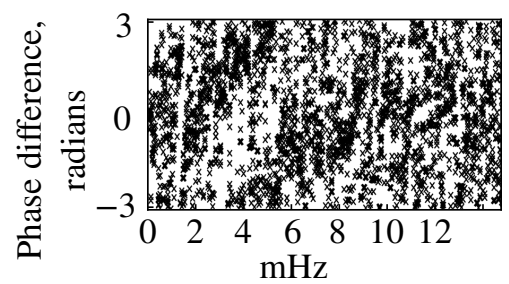

Figure 6: Time series No.4. The FFT phase difference of LOS-velocity oscillations $\left(V_{S i I}-V_{H e I}\right)$ for each facula element along the entrance slit and for a specific frequency.

$2^{\prime \prime}$ along the slit and is the longest phase lag detected for this time series. A lowest lag value of $40 \mathrm{~s}$ was measured for another spatial point along the slit. Table 1 presents these values as $40-87$ s for series No. 4. Similarly, the lag values are given for other time series. For the majority of the time series, the LOS velocity power spectrum in the Si I $10827 \AA$ line is very similar to that in the He I $10830 \AA$ line.

To diagnose the wave propagation, one can use a phase difference plot (Lites, 1992). Figure6 shows such a plot for time series No.4. Each " $\mathrm{x}$ " marks the phase difference of the LOS-velocity oscillations $\left(V_{S i I}-V_{H e I}\right)$ for each facula element along the entrance slit and particular frequency. Weak indications of propagating oscillations can be seen at $3 \mathrm{mHz}$, where one can see a jump in the phase difference. The time delay between the oscillations of these frequencies can be roughly estimated as $66 \mathrm{~s}$. This value is within the range between the lowest and highest values of the phase lags detected by direct comparison of the filtered LOS-velocity signals (Table 1, series No.4).

Line-of-sight velocity signals were obtained from different spectral lines formed at different heights within the solar atmosphere. The phase speed can be determined for an upward propagating wave. For Si I $10827 \AA$ and He I $10830 \AA$ the height difference is $1500 \mathrm{~km}$ (Avrett, Fontenla, and Loeser, 1994; Bard and Carlsson, 2008; Centeno, Collados, and Trujillo Bueno, 2009), see Table 3. If we observe the waves that travel upward at the photospheric sound speed $\left(4-6 \mathrm{~km} \mathrm{~s}^{-1}\right)$, then the lag is to be about $300 \mathrm{~s}$. There are no such lag values in our measurements (see Table 1). The 50-100s lags correspond to a speed of $15-30 \mathrm{~km} \mathrm{~s}^{-1}$. This is much higher than the speed of sound.

In contrast to the Si I $10827 \AA$ and He I $10830 \AA$ pair, power spectra for facular oscillations derived from the Fe I $6569 \AA$ and $\mathrm{H} \alpha 6563 \AA$ lines differ 

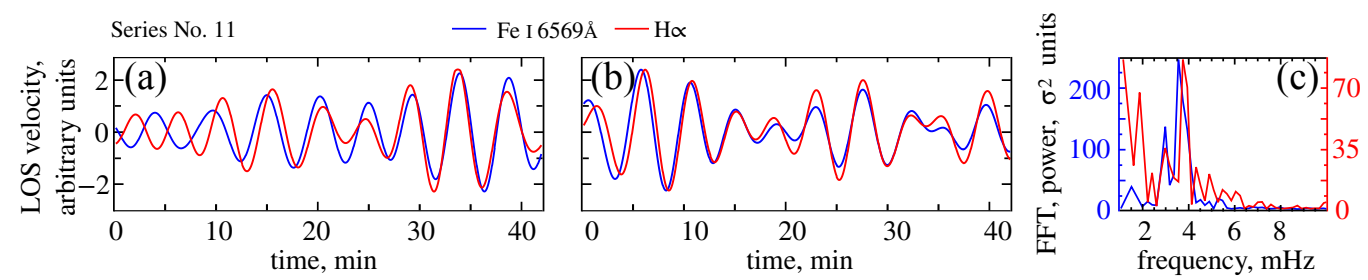

Figure 7: Time series No.11. Filtered LOS-velocity signals for two positions on the slit (a) and (b), 3-4 mHz filtration band. (c) Power spectra for the original signals shown in (a). Photospheric signal Fe I $6569 \AA$ - blue line; chromospheric signal $\mathrm{H} \alpha 6563 \AA$ - red line.

significantly in the low-frequency $(1-2 \mathrm{mHz})$ and high-frequency $(5-7 \mathrm{mHz})$ regions. The power of chromospheric oscillations at $1-2 \mathrm{mHz}$ frequencies obtained in $\mathrm{H} \alpha$ is often comparable to or exceeds the power of five-minute oscillations. A similarity between the spectra is observed only in the central part of the range $(2.5-4.5 \mathrm{mHz})$. The oscillation power in this spectral range is considerably higher than the noise level (Figure 7 (c)). The highest cross-correlation for the signals in Figure 7 (a) corresponds to an $8 \mathrm{~s}$ negative lag. On the other hand, the 10-20 minute range clearly shows a positive delay. The signals for another slit position of the same time series are shown in Figure 7 (b). The delay for the first wave train is $20 \mathrm{~s}$. The second wave train shows no delay between the photosphere and the chromosphere. The other time series obtained in these lines show a similar behaviour of the photospheric and chromospheric signals. The Fe I $6569 \AA$ line formation depth is $150-200 \mathrm{~km}$ (Parnell and Beckers, 1969) and that of $\mathrm{H} \alpha 6563 \AA$ is 1550-2000 (Vernazza, Avrett, and Loeser, 1981; Leenaarts, Carlsson, and Rouppe van der Voort, 2012). The longest 71s lag detected corresponds to a phase speed of about $21 \mathrm{~km} \mathrm{~s}^{-1}$.

The character of the oscillations detected in Si I $8536 \AA$ and Ca II $8542 \AA$ is similar to that observed in Fe I $6569 \AA$ and $\mathrm{H} \alpha 6563 \AA$. The first wave train in the photosphere differs notably from that in the chromosphere (Figure $8(\mathrm{a})$ ). If we assume that we observe the same wave train at both levels, the estimated phase delay is $48 \mathrm{~s}$. The second wave train is clearly identified at both levels and the chromospheric signal lags behind the photospheric signal by $30 \mathrm{~s}$. The third wave train shows an ambiguous delay that gradually changes from a slightly negative to a positive one. At the same time, oscillations in the five-minute band dominate at both levels, and their power is significantly 

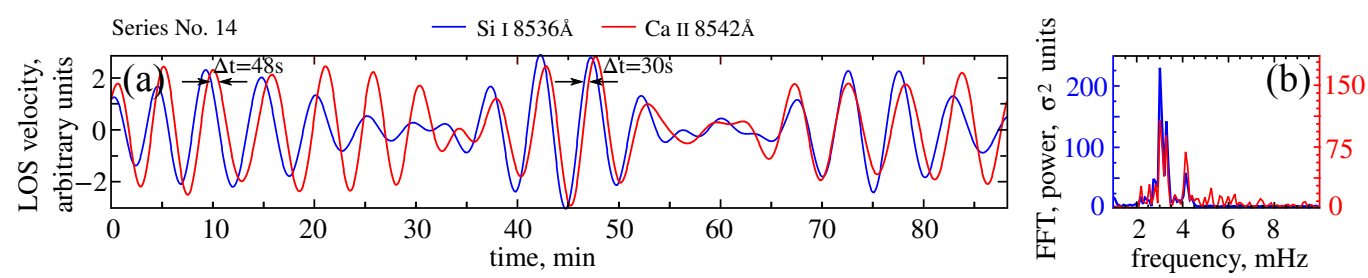

Figure 8: Time series No. 14. (a) Photospheric and chromospheric LOSvelocity signals filtered in the $2.5-3.5 \mathrm{mHz}$ band. (b) Corresponding power spectra of the LOS-velocity signals for two atmospheric levels. Photosphere Si I $8536 \AA$ - blue line; chromosphere Ca II $8542 \AA$ - red line.
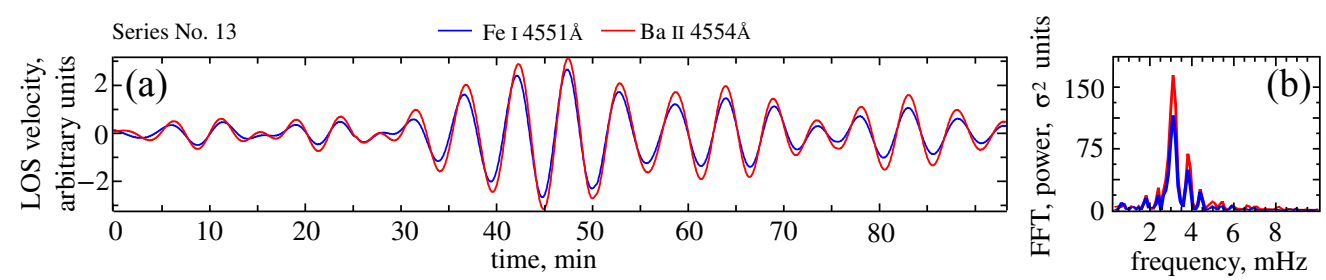

Figure 9: Time series No. 13: (a) LOS-velocity signals filtered in the 2.5-3.5 $\mathrm{mHz}$ band. (b) Corresponding power spectra of the LOS-velocity signals for two atmospheric levels. Photosphere Fe I $4551 \AA$ - blue line; temperature minimum Ba II $4554 \AA$ - red line. 
higher than the noise level (Figure 8 (b)). The estimated formation depth of the Ca II $8542 \AA$ line is within the $1200-1500 \mathrm{~km}$ range (Mein and Mein. 1980; Leenaarts et al., 2009). With a $1000 \mathrm{~km}$ height difference, this roughly corresponds to a lowest phase speed of $20-30 \mathrm{~km} \mathrm{~s}^{-1}$, which is much higher than the speed of sound in the photosphere.

In this context it is interesting to analyse the situation for the solar atmospheric levels that are close in height. For example, Fe I $4551 \AA$ and Ba II $4554 \AA$ provide us with such an opportunity. The core of the BaII $4554 \AA$ line forms at $640 \mathrm{~km}$ (Shchukina, Olshevsky, and Khomenko, 2009). The depth of the Fe I $4551 \AA$ line formation is $140 \mathrm{~km}$ (Gurtovenko and Kostyk, 1989). Hence, the maximal height difference is $500 \mathrm{~km}$. As can be seen from Figure 9, the signals for both levels are almost identical, except for an $8 \mathrm{~s}$ time lag and the amplitude difference by a factor of 2 . The whole facula (average over the slit) demonstrates the same behaviour. The longest $10 \mathrm{~s}$ lag (see Table 1) corresponds to a phase speed of $50 \mathrm{~km} \mathrm{~s}^{-1}$.

\subsection{Sunspots}

Waves in sunspots have been a matter of debate since the 1970s. In contrast to the faculae, three-minute oscillations dominate in the umbra sunspot chromosphere. The measured phase delays are still difficult to interpret (e.g. Lites (1992)). In the observations reported by different authors three-minute oscillations manifest themselves as standing waves or as upward-propagating waves. Lites and Thomas (1985) found that three-minute oscillations of the LOS velocity in the photosphere have the character of vertical standing waves. The early research (Uexküell, Kneer, and Mattig, 1983) estimated the wave's upward-propagating speed to be $10-25 \mathrm{~km} \mathrm{~s}^{-1}$, depending on the frequency. Giovanelli, Harvey, and Livingston (1978) measured the lag between photospheric and chromospheric oscillations to be about $26 \mathrm{~s}$ (Fe I $5233 \AA-\mathrm{H} \alpha$ pair). Kentischer and Mattig (1995) reported a 40 s time lag between the intensity signals of the $\mathrm{H} \alpha$ and Ca II K lines. This lag corresponds to a vertical phase speed of $7 \mathrm{~km} \mathrm{~s}^{-1}$. Kobanov et al. (2011a) determined a non-constant phase delay, changing from 20 to 140 s (Fe I $6569 \AA-\mathrm{H} \alpha$ lines). A comparison of the optical and radio observations showed a time delay of $50 \mathrm{~s}$ of the radio signal with respect to the optical signal (Abramov-Maximov et al., 2011). Long $\sim 300 \mathrm{~s}$ phase delays (low propagation speed) for the Si I $10827 \AA$ and He I $10830 \AA$ pair were reported by Centeno, Collados, and Trujillo Bueno (2009). 

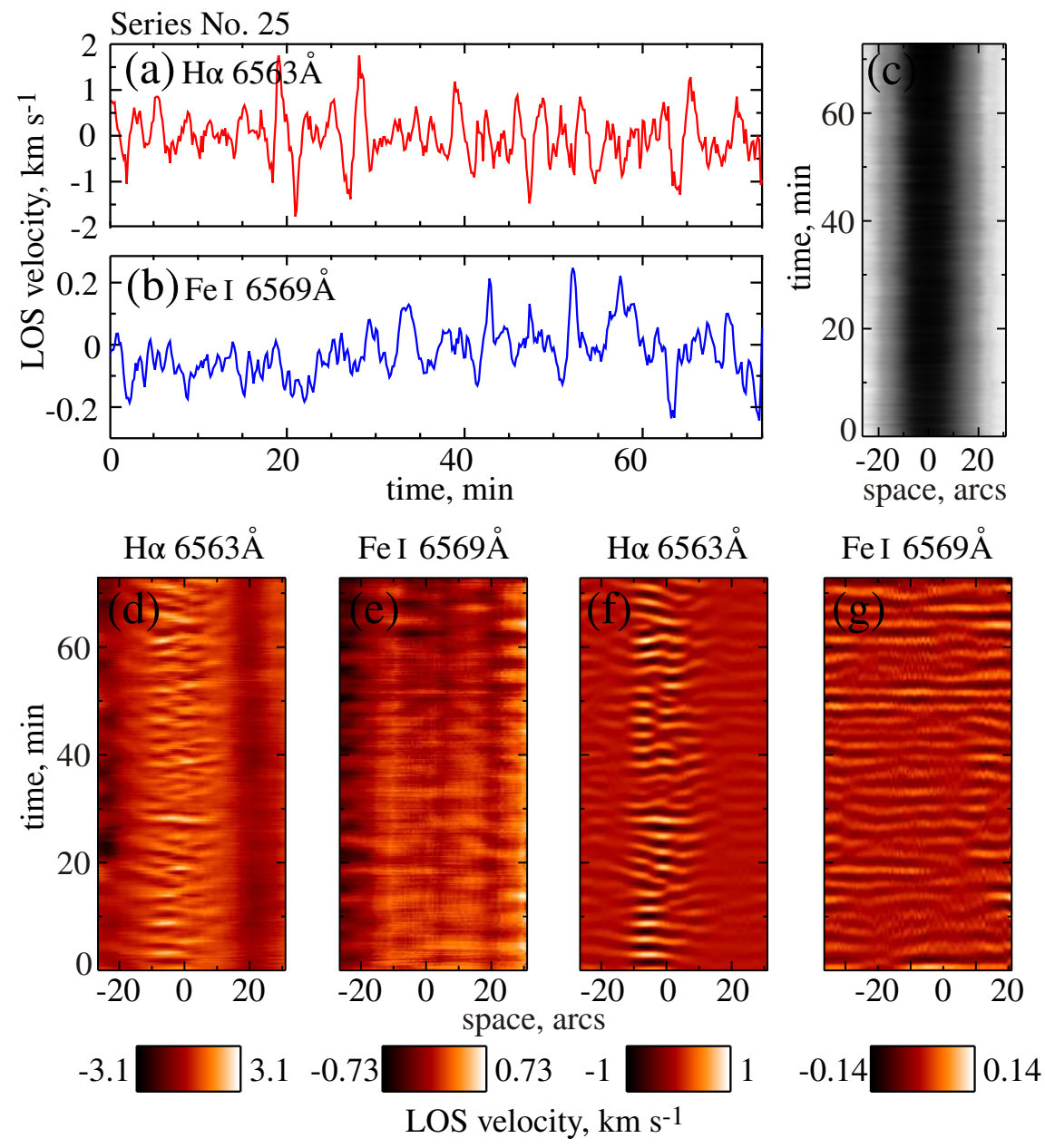

Figure 10: Time series No. 25. Overview of the data. Panels (a) and (b): unfiltered LOS-velocity signals for umbra centre slit position. Panel (c): intensity signal in continuum; (d) and (e): unfiltered diagrams of the LOSvelocity signals for the whole slit; (f) and (g): corresponding diagrams after the wavelet filtration $(4.6-7.2 \mathrm{mHz}$ band). 

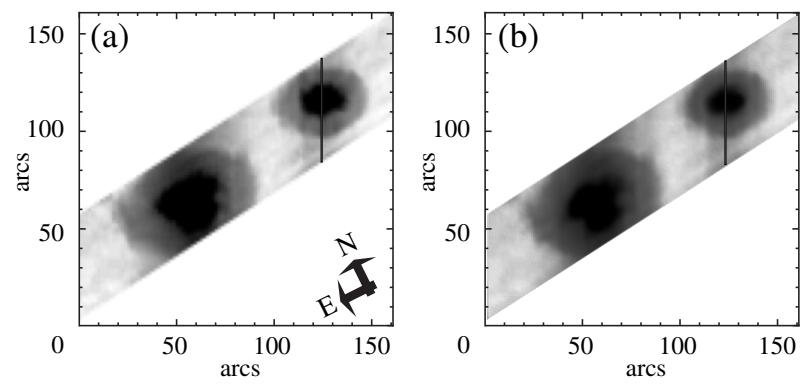

Figure 11: Overview scan of the active region that is observed in time series No. 20. Panel (a): before the time sequence and (b) after the time sequence.

(a)

(b)

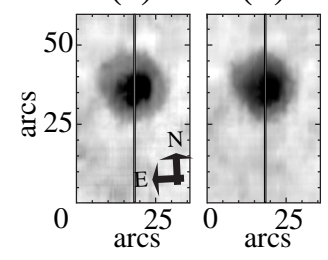

Figure 12: Overview scan of the active region that is observed in time series No. 16. Panel (a): before the time sequence and (b) after the time sequence.

(a) Stokes I, Si I

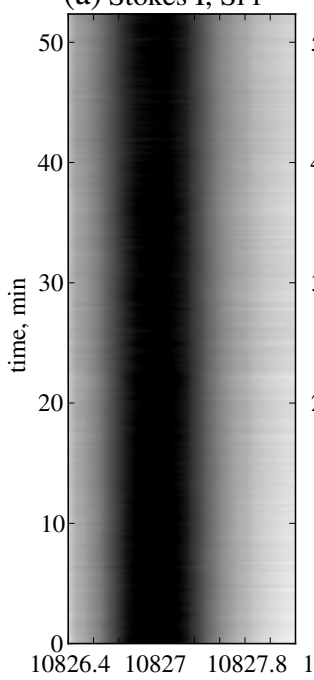

(b) Stokes I, He I

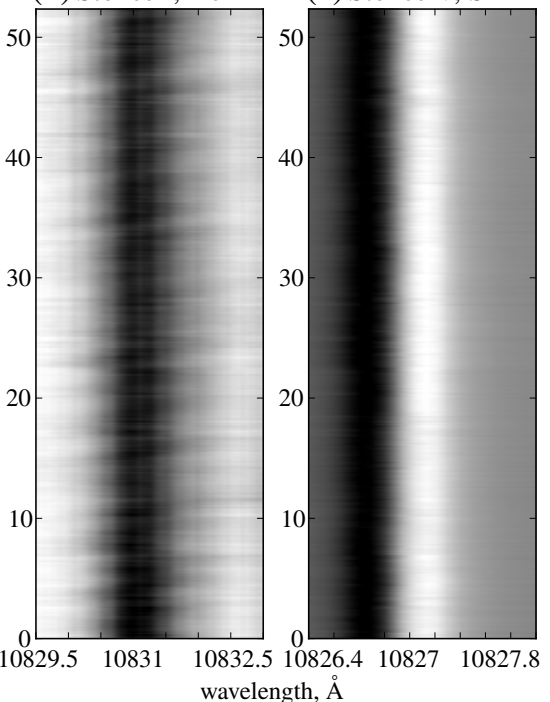

(d) Stokes V, He I

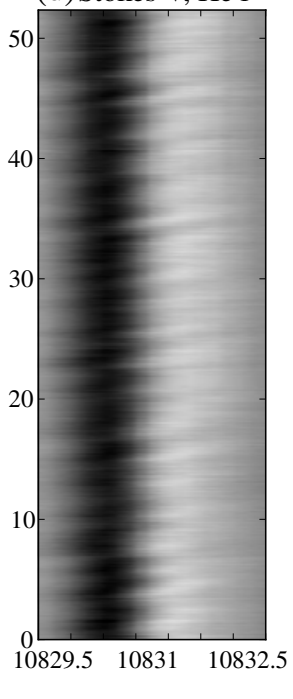

Figure 13: Time series No. 16. Temporal evolution of Stokes I and Stokes V for Si I $10827 \AA$ (a) and (c) and He I $10830 \AA$ (b) and (d). 


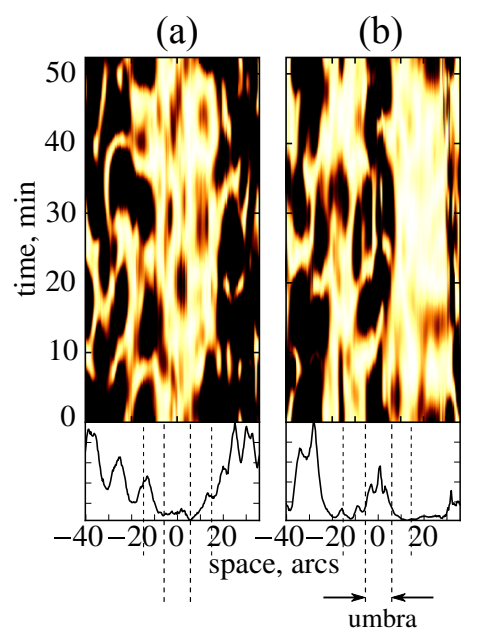

Figure 14: Time series No. 16. Power distribution of the three-minute oscillations for the umbral centre (a) photosphere and (b) chromosphere. Dashed lines denote the penumbra boundaries.

Here we present delays measured for 17 time series (12 sunspots, Table2). As an example, Figure 10 presents the data for time series No. 25. The sunspot position is stable throughout the whole time series (Figure 10 (c)). Panels (a) and (b) demonstrate unfiltered LOS-velocity signals for the umbra centre. Unfiltered space-time diagrams of the LOS-velocity signals for the whole slit can be found in panels (d) and (e). The corresponding diagrams after wavelet filtration $(4.6-7.2 \mathrm{mHz}$ band) are also provided (panels (f) and $(\mathrm{g})$ ). When possible, the time series were accompanied by the overview scan of the observed spot. Figure 11 shows an example of such a scan for time series No. 20 (continuum intensity). The spectrograph slit position is marked by the vertical line. In some time series a slit-jaw image taken through an $\mathrm{H} \alpha$ filter was used to simultaneously monitor the target position. The overview scan along with the Stokes I and V diagrams for the sunspot series No. 16 analysed below are shown in Figures 12 and 13 , respectively.

Three-minute photospheric oscillations in the spot umbra are suppressed compared to the neighbouring regions, whereas the chromospheric oscillations are enhanced. This is valid not only for the Fe I $6569 \AA-\mathrm{H} \alpha$ lines (Kobanov et al. 2011a), but also for the Si I $10827 \AA$ - He I $10830 \AA$ pair (see Figure 14). The distribution of the three-minute oscillation power spectrum across the slit in Figure 14 (a) has the lowest value in the umbral photo- 


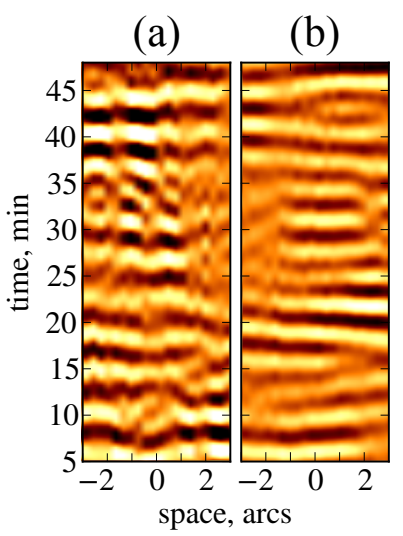

Figure 15: Time series No. 16. Space-time diagrams of LOS-velocity signals (Stokes-V zero-crossing, $4.6-5.6 \mathrm{mHz}$ filtration) for (a) the photosphere and (b) the chromosphere.

sphere. The chromospheric signal is highest at the same slit position. The filtration was performed for the $4.6-5.6 \mathrm{mHz}$ band. Series $19-25$ obtained in the Fe I $6569 \AA-\mathrm{H} \alpha$ lines show the power of the photospheric three-minute oscillations to be slightly higher than the noise level (exceeds $3 \sigma^{2}$ level). For most of these series it was not possible to find a good correspondence between the three-minute oscillations in the photosphere and the chromosphere. The cross-correlation between the signals is below 0.3 . The identification of wave trains at both levels was complicated. For these cases the "Lag" column of Table 2 contains a dash for the corresponding series.

The Si I $10827 \AA$ line is formed higher in the solar atmosphere than that of Fe I $6569 \AA$. The photospheric three-minute LOS-velocity oscillation power of the former is considerably higher than the power detected in the latter (see Figure $17(\mathrm{~b})$ and $(\mathrm{d}))$ ). In this case it is possible to identify wave trains at both atmospheric levels for the Si I $10827 \AA$ - He I $10830 \AA$ pair. LOSvelocity signals, filtered in the $4.6-5.6 \mathrm{mHz}$ band, are shown in Figure 15 (a) and (b). The highest cross-correlation of 0.65 between the two images can be achieved by a 51-s backward shift of the He I $10830 \AA$ image. The unfiltered LOS-velocity signals exhibit photospheric oscillations in the five-minute band (Figure 16 (a)) and the chromospheric oscillations in the three-minute band (Figure 16 (b)). The signals are shown for the single position along the slit $-2^{\prime \prime}$ away from the umbra centre. Figure 17 (a) and (c) presents these signals filtered to reveal the three-minute oscillations. The upper panels 

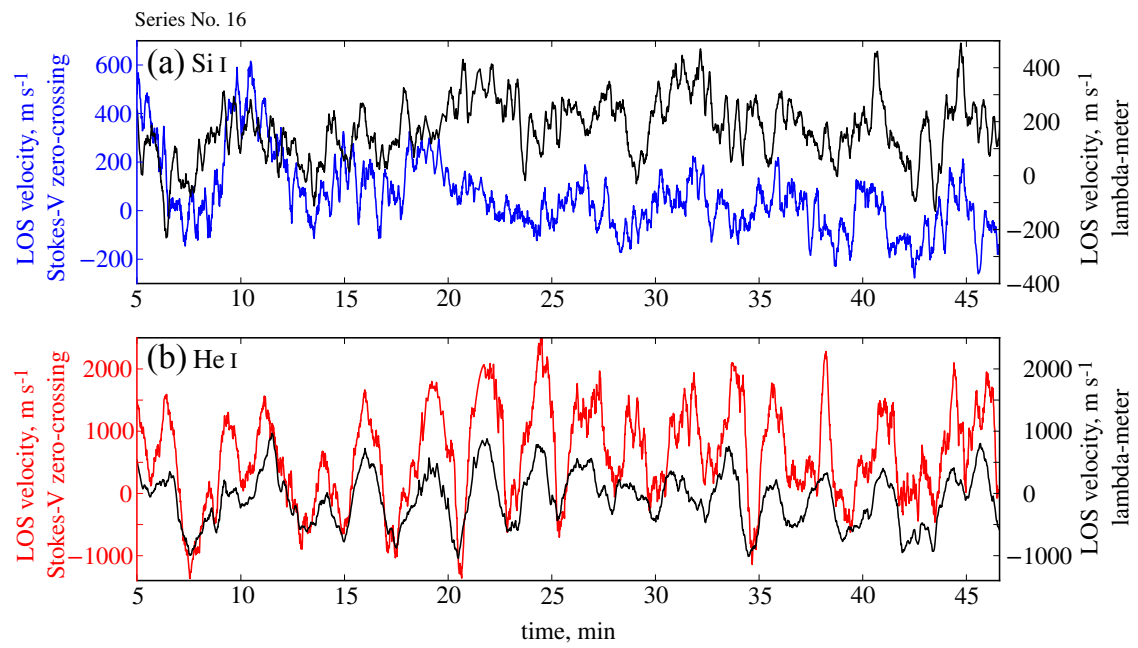

Figure 16: Time series No.16. The area corresponds to $1^{\prime \prime}$ along the slit. The unfiltered LOS velocities derived with the lambda-meter technique (black lines) and with tracking Stokes-V zero-crossing (blue and red): (a) Si I $10827 \AA$, (b) He I $10830 \AA$.

represent the results derived from the lambda-meter technique, the bottom panels depict the signals calculated by tracking Stokes-V zero-crossing. The right panels show the corresponding power spectra of the original (unfiltered) signals. The wavelet filtration was performed within the $4.6-5.6 \mathrm{mHz}$ range, where the three-minute oscillation power is higher than the noise level for all four signals (Figure $17(\mathrm{~b})$ and (d)). One can see two wave trains in Figure 17 (a) and (c). The signals are not identical, but differ to some extent. The wave packet in the chromosphere can significantly diverge from its photospheric counterpart. This is common in the analysed sunspots. For both techniques (lambda-meter and tracking Stokes-V zero crossing, Figure 17) the time delay between the photosphere and chromosphere varies, but it does not exceed $100 \mathrm{~s}$. The variations can be caused by several factors, including the presence of the fine structure in the umbra, or because we used different parts of the spectral line profile in two techniques. The longest time delay between the photosphere and chromosphere in Figure 17 is 64s. An example for series No. 17 in Figure 18(a) shows a negative time delay of -20 s. The $1000 \mathrm{~km}$ height difference and the $64-110 \mathrm{~s}$ lag (see Table 2 series No. $15-$ 18) correspond to the phase speed of $9-15 \mathrm{~km} \mathrm{~s}^{-1}$. 

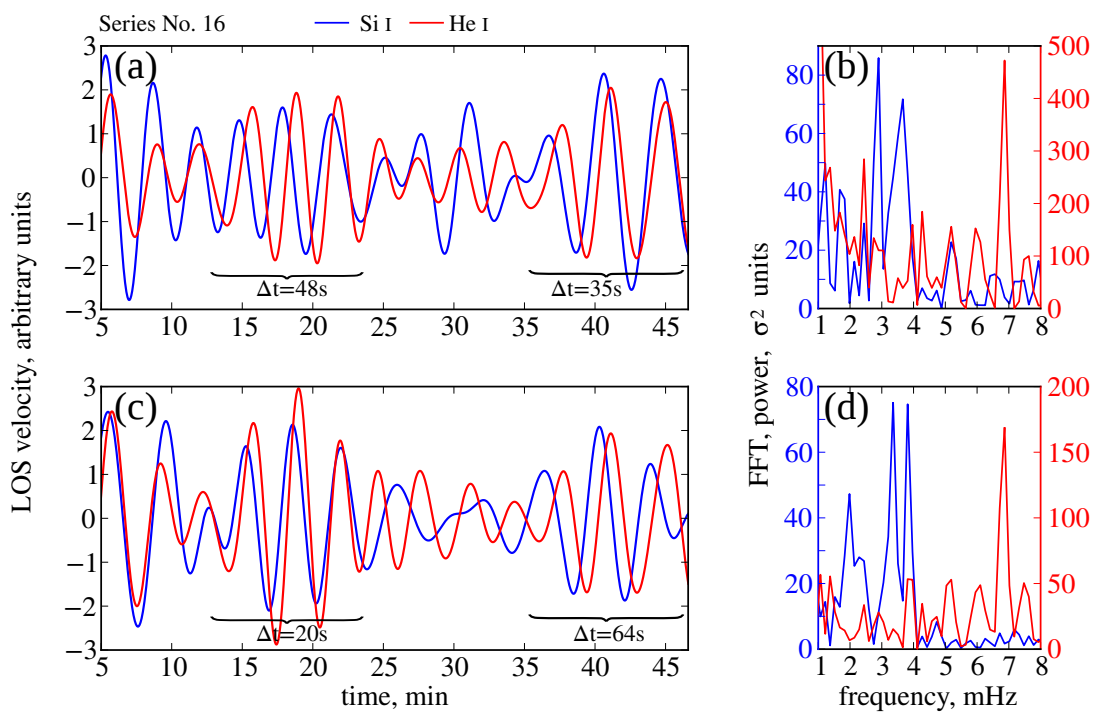

Figure 17: Time series No. 16. LOS-velocity signals $(4.5-5.6 \mathrm{mHz}$ filtration band) derived with (a) the lambda-meter technique and (c) with Stokes-V zero-crossing. The LOS-velocity power spectra for two atmospheric levels, derived witha (b) the lambda-meter technique and (d) with Stokes-V zerocrossing. Photosphere - Si I $10827 \AA$, blue line; chromosphere - He I $10830 \AA$, red line. The position along the slit is the same as for Figure 16.

The LOS-velocity oscillation phase difference $\left(V_{S i I}-V_{H e I}\right)$ for each umbra element along the entrance slit and for each specific frequency shows weak indications of propagating oscillations in the $5-6 \mathrm{mHz}$ band (Figure 19, time series No. 16). The phase delays between the photospheric and chromospheric oscillations of these frequencies deduced from these plots are (a) $50 \mathrm{~s}$ and (b) $80 \mathrm{~s}$.

At the Fe I $6569 \AA$ - H $\alpha$ line heights, the correspondence between the photosphere and the chromosphere is less prominent than for Si I $10827 \AA$ - He I $10830 \AA$. One can hardly find a correspondence between the two signals in Figure 20. The power of the photospheric signal of the three-minute band is slightly higher than the $3 \sigma^{2}$ level. The same is true for the other time series obtained in the Fe I $6569 \AA-\mathrm{H} \alpha$ lines.

For series obtained in Si I $8536 \AA$ - Ca II $8542 \AA$ a similar ambiguity between the photospheric and chromospheric heights was found, except for one 


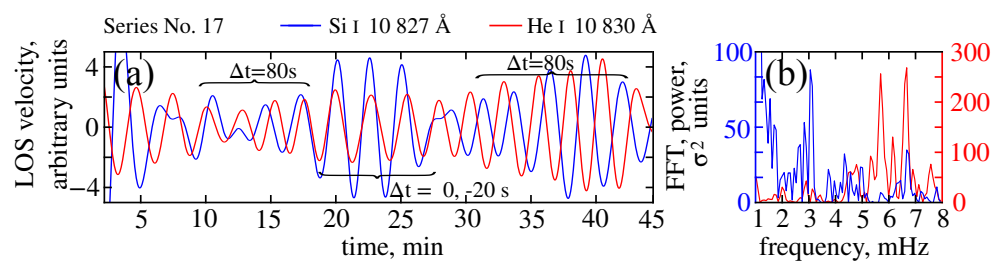

Figure 18: Time series No. 17, umbral region of $1^{\prime \prime}$. (a) LOS velocity (Stokes$\mathrm{V}$ zero-crossing) filtered in the $6.0-7.0 \mathrm{mHz}$ band. (b) The LOS-velocity power spectra for two atmospheric levels. Photosphere - Si I $10827 \AA$, blue line; chromosphere - He I $10830 \AA$, red line.

(a)

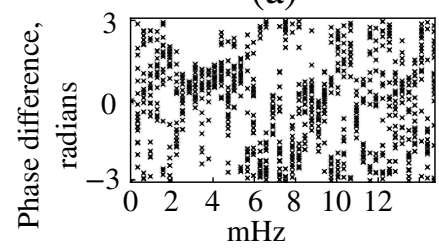

(b)

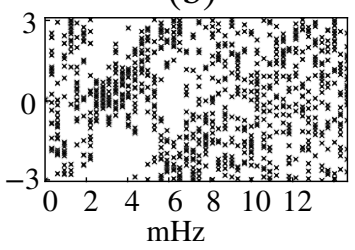

Figure 19: Time series No.16. The FFT phase difference of LOS-velocity oscillations $\left(V_{S i I}-V_{H e I}\right)$ for each umbra element and specific frequency. LOS velocity derived from (a) lambda-meter technique; (b) Stokes V-zero crossing position.

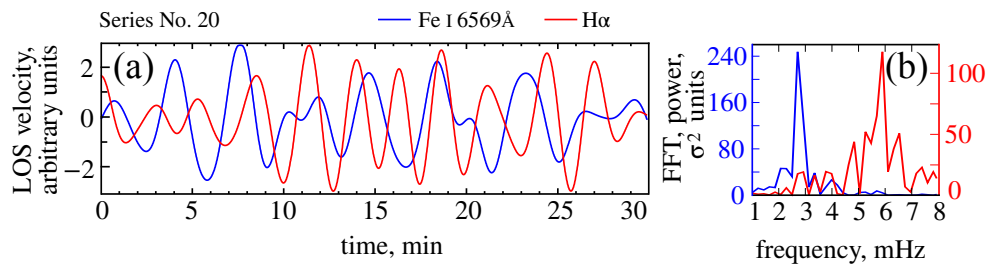

Figure 20: Time series No. 20, umbral region of $1^{\prime \prime}$. (a) LOS velocity (lambdameter technique) filtered in the $4.6-5.6 \mathrm{mHz}$ band. (b) The LOS-velocity power spectra at two atmospheric levels. Photospheric signal Fe I $6569 \AA-$ blue line; chromospheric signal $\mathrm{H} \alpha 6563 \AA$ - red line. 

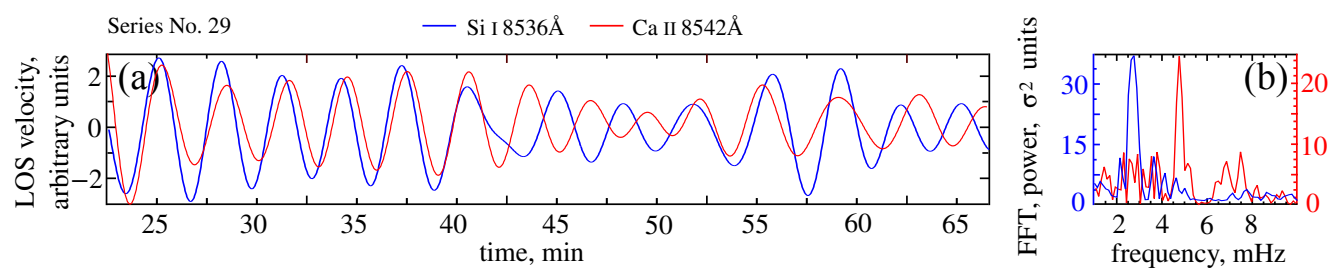

Figure 21: Time series No. 29: (a) filtered photospheric and chromospheric LOS-velocity signals $(4.5-5.5 \mathrm{mHz})$. (b) The corresponding LOS-velocity power spectra for two atmospheric levels. Photosphere Si I $8536 \AA$ - blue line; chromosphere - Ca II $8542 \AA$ - red line.
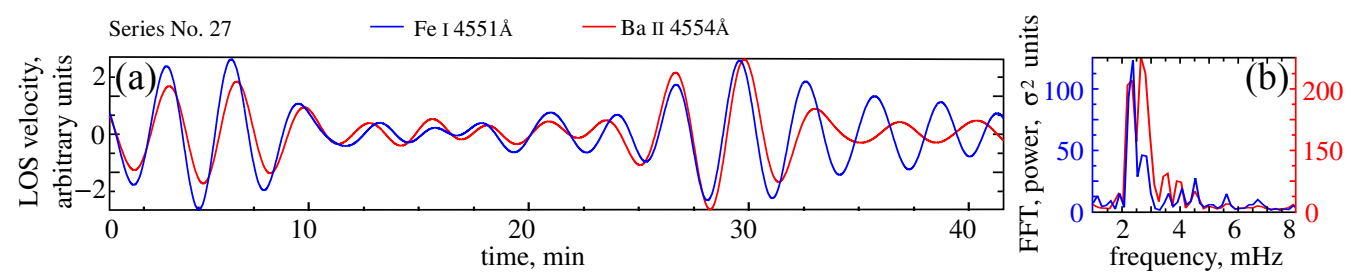

Figure 22: Time series No. 27, umbral region of $1^{\prime \prime}$. (a) LOS velocities filtered in the $5-6 \mathrm{mHz}$ band. (b) The LOS-velocity power spectra at two atmospheric levels. Photosphere Fe I $4551 \AA$ - blue line; temperature minimum Ba II $4554 \AA$ - red line.

series discussed below, where it was possible to determine a time delay. The power spectra for the chromosphere and photosphere in Figure 21 (b) are very different. Nevertheless, both signals have a significant power within the $4.5-5.5 \mathrm{mHz}$ and $7-8 \mathrm{mHz}$ bands, and there ia a wave train correspondence (Figure 21 (a)). The lag between the filtered signals $(4.5-5.5 \mathrm{mHz})$ in Figure 21 (a) is $21 \mathrm{~s}$. This corresponds to the upward-propagating wave at a speed of $43 \mathrm{~km} \mathrm{~s}^{-1}$, if the height difference is $900 \mathrm{~km}$. The other series (Table2, series No. 30, 31) do not reveal any correspondence between wave trains at the two levels.

The situation is totally different for the time series obtained in Fe I $4551 \AA$ and BaII $4554 \AA$ (formation heights are 140 and $640 \mathrm{~km}$, respectively). As for the faculae, there is almost a perfect correspondence between wave trains at two heights for the most sunspot umbra slit positions (Figure 22). For the signals shown in Figure 22 the lag is about $10 \mathrm{~s}$. With a $500 \mathrm{~km}$ height difference, this lag corresponds to a phase speed of $50 \mathrm{~km} \mathrm{~s}^{-1}$. 


\section{Discussion and Conclusions}

We analysed LOS-velocity oscillations at different pairs of solar atmosphere levels for faculae and sunspots. It is rare that the wave trains correspond directly for sunspots. When the correspondence was obvious, we measured a time delay that appeared to be short on average. The short delay we found may indicate that the phase speed is significantly higher than the commonly used value $4-6 \mathrm{~km} \mathrm{~s}^{-1}$ for the photosphere. The signal produced with the lambda-meter technique may be affected by the non-magnetic elements, whereas the signal yielded by tracking the null of the Stokes-V profile is formed only by the part where the magnetic field is present. Obviously, this difference is very small for the sunspot umbra, where the magnetic filling factor is close to 1 . For two levels of the solar atmosphere that are close in height $(\Delta h=500 \mathrm{~km}$, Fe I $4551 \AA$ and BaII $4554 \AA)$ the signals agree much more often than for the other analysed levels where the height difference is significantly higher. However, the measured time delays also correspond to the high propagation velocities. An ambiguous results for the Fe I $6569 \AA-$ $\mathrm{H} \alpha 6563 \AA$ pair is likely due to the fact that at a moderate spatial resolution of $1.5^{\prime \prime}$ the three-minute oscillations at the Fe I $6569 \AA$ formation height are extremely weak. It is possible that a high spatial resolution will allow one to detect domains with more powerful oscillations at that level.

The interpretation of the lags and phase speeds critically depends on the assumed line formation heights. Most of them are derived from quiet-Sun conditions and are not valid in magnetic regions, either in faculae or sunspots. To compare our results with those from other authors, we intentionally used the same height difference values as they did. We can assume that the height scale is significantly reduced in the presence of the magnetic field. In this case, the similarity between the oscillations detected in different lines is to be like the one revealed in the Fe I $4551 \AA$ and Ba II $4554 \AA$ pair. However, the analysis performed for different pairs showed such a similarity neither for the sunspots nor for the faculae under investigation.

We assume that within the observed cavity standing and propagating waves co-exist, therefore the measured phase lag depends on the contribution of each of the components. Their rate is determined by the resonator boundary penetrability degree, which in real conditions changes following local changes in temperature, pressure, and magnetic field. The "chevrons"

on space-time diagrams clearly visible above spot umbra in the $5.5-7 \mathrm{mHz}$ band (Kobanov, Kolobov, and Makarchik, 2006) may be considered as evi- 
dence of the resonator's upper boundary transparency. The short delay raises the question of the oscillations origin. Our results support the hypothesis of a chromospheric resonator in sunspots (Zhugzhda, Locans, and Staude, 1985; Botha et al., 2011), rather than the hypothesis of linear wave propagation with sound speed from the photosphere to the chromosphere (Centeno, Collados, and Trujillo Bueno, 2009).

In the case of faculae the situation is different. For most of the faculae, the five-minute oscillation periods are dominant either in the photosphere or in the chromosphere. An ambiguous behaviour of the time lags can be explained in the following way. Inclined magnetic flux tubes that contribute to the signal for the lower height levels can move beyond the aperture range at the higher levels, and vice-versa, other flux tubes can fall into the aperture. We discussed this possibility in Kobanov et al. (2011b). It would be useful to analyse velocity signals obtained by both techniques (Stokes-V zero-crossing and lambda-meter). The magnetic filling factors are significantly less than one in the facular regions at the photospheric level (Martinez Pillet, Lites, and Skumanich, 1997; Muglach and Solanki, 1992). This would lead to different results obtained by both techniques for the photosphere, while in the chromosphere the expansion of the flux tubes might smooth this difference (see Figure 3, Khomenko et al. (2008)). However, these measurements allow one to determine the non-magnetised component contribution (e.g. surrounding $\mathrm{p}$ modes). In addition, the rare correspondence between the signals at two heights may be explained by the dispersive properties of the medium that smear the wave packet significantly.

Observations made simultaneously for many lines formed at different heights would certainly clarify the problem. The spectral line set presented here seems quite appropriate for this task.

Acknowledgements. This study was supported in part by the RFBR research project No.: 12-02-33110 mol_a_ved and the Grant of the President of the Russian Federation No.: MK-497.2012.2, Russian Federation Ministry of Education and Science state contract No.: 14.518.11.7047 and agreement No.: 8407. 


\section{References}

Abramov-Maximov, V.E., Gelfreikh, G.B., Kobanov, N.I., Shibasaki, K., Chupin, S.A.: 2011, Multilevel Analysis of Oscillation Motions in Active Regions of the Sun. Solar Phys. 270, 175 -189. doi:10.1007/s11207-011-9716-7.

Avrett, E.H., Fontenla, J.M., Loeser, R.: 1994, Formation of the Solar 10830 Angstrom Line. In: D. M. Rabin, J. T. Jefferies, \& C. Lindsey (ed.) Infrared Solar Physics, IAU Symposium 154, 35.

Balthasar, H.: 2003, Oscillations in Sunspots observed in the Near Infrared. Solar Phys. 218, 85-97. doi:10.1023/B:SOLA.0000013028.11720.0d.

Balthasar, H., Muglach, K.: 2010, The three-dimensional structure of sunspots. II. The moat flow at two different heights. Astron. Astrophys. 511, A67. doi:10.1051/0004-6361/200912978.

Bard, S., Carlsson, M.: 2008, Constructing Computationally Tractable Models of Si for the $1082.7 \mathrm{~nm}$ Transition. Astrophys. J. 682, 1376-1385. doi: $10.1086 / 589910$.

Beckers, J.M., Tallant, P.E.: 1969, Chromospheric Inhomogeneities in Sunspot Umbrae. Solar Phys. 7, 351.

Bloomfield, D.S., Lagg, A., Solanki, S.K.: 2007, The Nature of Running Penumbral Waves Revealed. Astrophys. J. 671, $1005-1012$. doi:10.1086/523266.

Botha, G.J.J., Arber, T.D., Nakariakov, V.M., Zhugzhda, Y.D.: 2011, Chromospheric Resonances above Sunspot Umbrae. Astrophys. J. 728, 84. doi: $10.1088 / 0004-637 X / 728 / 2 / 84$

Centeno, R., Collados, M., Trujillo Bueno, J.: 2009, Wave Propagation and Shock Formation in Different Magnetic Structures. Astrophys. J. 692, 1211-1220. doi: $10.1088 / 0004-637 X / 692 / 2 / 1211$.

Christopoulou, E.B., Georgakilas, A.A., Koutchmy, S.: 2000, Oscillations and running waves observed in sunspots. Astron. Astrophys. 354, 305-314.

Finsterle, W., Jefferies, S.M., Cacciani, A., Rapex, P., Giebink, C., Knox, A., Dimartino, V.: 2004, Seismology of the solar atmosphere. Solar Phys. 220, 317 - 331. doi:10.1023/B:SOLA.0000031397.73790.7b. 
Georgakilas, A.A., Christopoulou, E.B., Koutchmy, S.: 2000, Oscillations and running waves observed in sunspots. II. Photospheric waves. Astron. Astrophys. 363, $306-310$.

Giovanelli, R.G.: 1972, Oscillations and Waves in a Sunspot. Solar Phys. 27, 71.

Giovanelli, R.G., Harvey, J.W., Livingston, W.C.: 1978, Motions in solar magnetic tubes. III - Outward wave propagation in sunspot umbras. Solar Phys. 58, 347 361.

Gurman, J.B.: 1987, Sunspot umbral oscillations in Mg II K. Solar Phys. 108, $61-75$.

Gurman, J.B., Leibacher, J.W., Shine, R.A., Woodgate, B.E., Henze, W.: 1982, Transition region oscillations in sunspots. Astrophys. J. 253, 939-948. doi:10.1086/159692.

Gurtovenko, E.A., Kostyk, R.I.: 1989, Fraunhofer spectrum and a system of solar oscillator strengths. Kiev Izdatel Naukova Dumka.

Hammerschlag, R.H., Zwaan, C.: 1973, An Efficient Wind Shield for the Protection of Telescopes. Pub. Astron. Soc. Pac. 85, 468. doi:10.1086/129487.

Kentischer, T.J., Mattig, W.: 1995, Oscillations above sunspot umbrae. Astron. Astrophys. 300, 539.

Khomenko, E., Centeno, R., Collados, M., Trujillo Bueno, J.: 2008, Channeling 5 Minute Photospheric Oscillations into the Solar Outer Atmosphere through Small-Scale Vertical Magnetic Flux Tubes. Astrophys. J. Lett. 676, L85-L88. doi: $10.1086 / 587057$.

Kobanov, N.I., Kolobov, D.Y., Makarchik, D.V.: 2006, Umbral Three-Minute Oscillations and Running Penumbral Waves. Solar Phys. 238, 231-244. doi: $10.1007 /$ s11207-006-0160-z.

Kobanov, N.I., Kolobov, D.Y., Sklyar, A.A., Chupin, S.A., Pulyaev, V.A.: 2009, Characteristics of oscillatory-wave processes in solar structures with various magnetic field topology. Astron. Rep. 53(10), 957-967. doi: $10.1134 /$ S1063772909100072.

Kobanov, N.I., Kolobov, D.Y., Chupin, S.A., Nakariakov, V.M.: 2011a, Height distribution of the power of 3-min oscillations over sunspots. Astron. Astrophys. 525, A41. doi:10.1051/0004-6361/200913533. 
Kobanov, N.I., Kustov, A.S., Pulyaev, V.A., Chupin, S.A.: 2011b, The role of faculae in wave-energy transfer to upper layers of the solar atmosphere: Observations. Astronomy Reports 55, 532-540. doi:10.1134/S1063772911050052.

Kolobov, D.Y., Kobanov, N.I., Grigoryev, V.M.: 2008, A Spectropolarimeter for Studying Solar Magnetic Fields. Instruments and Experimental Techniques 51(1), 124-129.

Leenaarts, J., Carlsson, M., Rouppe van der Voort, L.: 2012, The Formation of the $\mathrm{H} \alpha$ Line in the Solar Chromosphere. Astrophys. J. 749, 136. doi:10.1088/0004$637 \mathrm{X} / 749 / 2 / 136$.

Leenaarts, J., Carlsson, M., Hansteen, V., Rouppe van der Voort, L.: 2009, ThreeDimensional Non-LTE Radiative Transfer Computation of the CA 8542 Infrared Line From a Radiation-MHD Simulation. Astrophys. J. Lett. 694, L128-L131. doi $10.1088 / 0004-637 X / 694 / 2 /$ L128.

Lites, B.W.: 1986, Photoelectric observations of chromospheric sunspot oscilla-

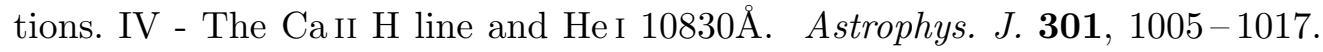
doi: $10.1086 / 163964$.

Lites, B.W.: 1992, Sunspot oscillations - Observations and implications. In: Thomas, J.H., Weiss, N.O. (eds.) NATO ASIC Proc. 375: Sunspots. Theory and Observations, $261-302$.

Lites, B.W., Chipman, E.G.: 1979, The vertical propagation of waves in the solar atmosphere. I - Observations of phase delays. Astrophys. J. 231, 570-588. doi $10.1086 / 157219$.

Lites, B.W., Thomas, J.H.: 1985, Sunspot umbral oscillations in the photosphere and low chromosphere. Astrophys. J. 294, 682-688. doi:10.1086/163338.

Martinez Pillet, V., Lites, B.W., Skumanich, A.: 1997, Active Region Magnetic Fields. I. Plage Fields. Astrophys. J. 474, 810. doi:10.1086/303478.

McAteer, R.T.J., Gallagher, P.T., Williams, D.R., Mathioudakis, M., Bloomfield, D.S., Phillips, K.J.H., Keenan, F.P.: 2003, Observational Evidence for Mode Coupling in the Chromospheric Network. Astrophys. J. 587, 806-817. doi: $10.1086 / 368304$

Mein, N., Mein, P.: 1980, Mechanical flux in the solar chromosphere. I - Velocity and temperature weighting functions for CaII lines. Astron. Astrophys. 84, 96-98. 
Moore, R.L., Tang, F.: 1975, Umbral oscillations and penumbral waves in H alpha. Solar Phys. 41, 81-88.

Muglach, K., Solanki, S.K.: 1992, Infrared lines as probes of solar magnetic features. I - A many-line analysis of a network region. Astron. Astrophys. 263, $301-311$.

Nagashima, K., Sekii, T., Kosovichev, A.G., Shibahashi, H., Tsuneta, S., Ichimoto, K., Katsukawa, Y., Lites, B., Nagata, S., Shimizu, T., Shine, R.A., Suematsu, Y., Tarbell, T.D., Title, A.M.: 2007, Observations of Sunspot Oscillations in G Band and CaII H Line with Solar Optical Telescope on Hinode. Pub. Astron. Soc. Japan 59, 631.

Nikulin, N.S., Severny, A.B., Stepanov, V.E.: 1958, The Solar Magnetograph of the Crimean Astrophysical Observatory. Krymskaia Astrofiz. Obs., Izvest. 19, $3-20$.

Parnell, R.L., Beckers, J.M.: 1969, The Interpretation of Velocity Filtergrams. I: The Effective Depth of Line Formation. Solar Phys. 9, 35-38. doi: $10.1007 /$ BF00145725.

Rayrole, J.: 1967, Contribution à l'étude de la structure du champ magnétique dans les taches solaires. Annales d'Astrophysique 30, 257.

Reznikova, V.E., Shibasaki, K., Sych, R.A., Nakariakov, V.M.: 2012, Three-minute Oscillations above Sunspot Umbra Observed with the Solar Dynamics Observatory/Atmospheric Imaging Assembly and Nobeyama Radioheliograph. Astrophys. J. 746, 119. doi:10.1088/0004-637X/746/2/119.

Rouppe van der Voort, L.H.M., Rutten, R.J., Sütterlin, P., Sloover, P.J., Krijger, J.M.: 2003, La Palma observations of umbral flashes. Astron. Astrophys. 403, 277 - 285. doi:10.1051/0004-6361:20030237.

Shchukina, N.G., Olshevsky, V.L., Khomenko, E.V.: 2009, The solar Ba II 4554 $\AA$ line as a Doppler diagnostic: NLTE analysis in 3D hydrodynamical model. Astron. Astrophys. 506, 1393-1404. doi:10.1051/0004-6361/200912048.

Sych, R., Zaqarashvili, T.V., Nakariakov, V.M., Anfinogentov, S.A., Shibasaki, K., Yan, Y.: 2012, Frequency drifts of 3-min oscillations in microwave and EUV emission above sunspots. Astron. Astrophys. 539, A23. doi:10.1051/00046361/201118271. 
Torrence, C., Compo, G.P.: 1998, A Practical Guide to Wavelet Analysis. Bull. Am. Meteo. Soc. 79, 61-78.

Uexküell, M.V., Kneer, F., Mattig, W.: 1983, The chromosphere above sunspot umbrae. IV - Frequency analysis of umbral oscillations. Astron. Astrophys. 123, $263-270$.

Vernazza, J.E., Avrett, E.H., Loeser, R.: 1981, Structure of the solar chromosphere. III - Models of the EUV brightness components of the quiet-sun. Astron. Astrophys. Suppl. 45, 635-725. doi:10.1086/190731.

Zhugzhda, I.D., Locans, V., Staude, J.: 1985, Oscillations in the chromosphere and transition region above sunspot umbrae - A photospheric or a chromospheric resonator? Astron. Astrophys. 143, 201-205.

Zirin, H., Stein, A.: 1972, Observations of Running Penumbral Waves. Astrophys. J. Lett. 178, L85. 